\title{
Choosing the Best Locomotion Mode in Reconfigurable Rovers
}

\author{
Carlos Jesús Pérez del Pulgar Mancebo ${ }^{1, *,+}$ (C) Pablo Romeo Manrique ${ }^{1,+}$, \\ Gonzalo Jesús Paz Delgado ${ }^{1,+}$, José Ricardo Sánchez Ibáñez ${ }^{1,+} \mathbb{D}$ and Martin Azkarate ${ }^{2} \mathbb{D}$ \\ 1 Department of Systems Engineering and Automation, Universidad de Málaga, Andalucía Tech, \\ 29071 Málaga, Spain \\ 2 European Space Agency (ESA-ESTEC), 2201 AZ Noordwijk, The Netherlands \\ * Correspondence: carlosperez@uma.es; Tel.: +34-951-952-324 \\ + These authors contributed equally to this work.
}

Received: 13 June 2019 ; Accepted: 17 July 2019; Published: 22 July 2019

\begin{abstract}
The use of autonomous rovers for planetary exploration is crucial to traverse long distances and perform new discoveries on other planets. One of the most important issues is related to the interaction between the rover wheel and terrain, which would help to save energy and even avoid getting entrapped. The use of reconfigurable rovers with different locomotion modes has demonstrated improvement of traction and energy consumption. Therefore, the objective of this paper is to determine the best locomotion mode during the rover traverse, based on simple parameters, which would be obtained from propioceptive sensors. For this purpose, interaction of different terrains have been modelled and analysed with the ExoTeR, a scale prototype rover of the European ExoMars 2020 mission. This rover is able to perform, among others, the wheel walking locomotion mode, which has been demonstrated to improve traction in different situations. Currently, it is difficult to decide the instant time the rover has to switch from this locomotion mode to another. This paper also proposes a novel method to estimate the slip ratio, useful for deciding the best locomotion mode. Finally, results are obtained from an immersive simulation environment. It shows how each locomotion mode is suitable for different terrains and slopes and the proposed method is able to estimate the slip ratio.
\end{abstract}

Keywords: rovers; locomotion modes; planetary exploration

\section{Introduction}

Exploring places beyond Earth, such as the surface of other planets, is key to unveiling some of the secrets of the universe. However, due to the current limitations in transporting humans to other planets, as well as the hostile conditions outside Earth, the use of automated vehicles-commonly known as rovers-arises as a practical solution. These vehicles are nowadays capable of carrying scientific instrumentation to many extraterrestial places. Nevertheless, the use of rovers entails a series of limitations and difficulties. On one hand, direct commanding is limited by the latency present in communications when the rover is placed on another planet. For example, any two-way messages from ground stations on Earth to a rover on the Martian surface can suffer a latency between 5 and $40 \mathrm{~min}$ [1]. This fact prevents the use of direct teleoperation. Thus, autonomous rovers are desired so as to maximise the scientific return from their exploration missions. On the other hand, other planets and satellites present extreme environments, where there are surfaces with multiple shapes and terrains with diverse composition. They present a challenge to the rover locomotion subsystems - any miscalculation in the planned traverse may result in the rover being harmed or even entrapped [2]. For this reason, both the availability of proper locomotion systems and the 
capability to make better use of them, depending on terrain conditions, are crucial to developing a fully autonomous rover.

Towards maximising rover mobility, a lot of research has been carried out with the aim of providing rovers the capability to traverse multiple types of terrain.

This is key to overcoming traversability problems as well as to optimise the traverse in a larger variety of terrains. This can be done by means of defining, for the same rover, various locomotion modes, that is, methods to interact with the terrain to make it advance. Depending on the dynamics of these modes, they will be more suitable for certain terrains than others. This is translated into the need of using rovers capable of reconfiguring their locomotion, instead of those restricted to a single locomotion mode. In this way, reconfigurable rovers can take the best out of their multiple locomotion capabilities to adapt better to a wider range of terrains. For example, the usual locomotion mode (called normal driving), based on driving with the rolling motion of the wheels, may be complemented by other modes such as the push-pull method introduced by Creager et al. [3], also called wheel-walking. This mode consists on making the vehicle deploy and retrieve its wheels, producing a motion that resembles the one used by inchworms. In this way, push-pull can compact soft terrains in those cases where usual driving would be inefficient or even useless, increasing the drawbar pull as result. Other authors [4] proposed the use of two new locomotion modes called rolling and peristaltic modes. The first one configures the rover to enhance the locomotion performance on slopes, and the second one performs a motion similar to worms, that is, moving the rover body ahead using the rover legs in a cyclic gait where each pair of wheels is moved meanwhile the other pair of wheels remains braced to the ground.

A more recent study by Fujiwara and lizuka [5] reinforces this statement, analyzing the augmentation in the resistance force of the terrain due to the soil being bulldozed. Furthermore, since the use of active articulated legs can also help to thrust rovers on loose soil, as demonstrated in Reference [6], the combination of them along with wheels introduces new possible locomotion modes [7-10]. Such combination, together with a rocker-bogie kinematic configuration, is present in the ExoTeR (Exomars Testing Rover). It is an experimental prototype, owned by the European Space Agency (ESA), that serves as a scaled version of the rover that is intended to be sent during the ExoMars 2020 mission [11]. One of its main particularities is the placement of motors on top of its legs, making the ExoTeR capable of deploying and retrieving its legs and augmenting, as result, its performance on loose soils as well as on certain inclined terrains [12]. Some simulations and real experiments about the real ExoMars rover were carried out in Reference [13]. In this paper, a wheel walking motion was introduced. It consisted on moving a pair of wheels (left and right wheel) from front to rear and vice versa. They demonstrated, in a simulation environment, that the rover was able to climb slopes up to $25^{\circ}$ using wheel-walking instead of $18^{\circ}$ with normal driving. The use of simulation environments to test and validate rover structures and performance have been widely used. As another example, apart from the previously mentioned paper, Thomas Thueer et al. [14] used the performance optimization tool (POT) to optimise the rover structure based on different parameters.

Although the benefits of employing reconfigurable rovers have been stated, the capability to better adapt to the existing terrain conditions in an autonomous way, that is, to decide the proper locomotion mode to use at each situation, demands the use of onboard sensory systems. In this way, the absence of prior knowledge about the extraterrestial environment [15] is compensated, making the rover react better to the surface changes and also to predict its features. For example, by means of the camera sensors the slip ratio can be determined $[16,17]$. Besides, the use of proprioceptive sensors enhances the characterization of the terrain, registering readings about inertia and vibrations [18]. Therefore, the use of sensors play an important role in the definition of vehicle-terrain parameters, which is enhanced with the combined use of dynamic terramechanic models [19]. In this way, the effectiveness of the locomotion mode employed could be determined by analysing the impact of these parameters on navigation measurements, such as the traversed distance and/or the power 
consumption. This approach offers a valid criteria that can be used by the rover to decide the best locomotion mode.

This paper provides two main novelties. The first is a deep study of two locomotion modes that would be used in reconfigurable rovers-normal driving and wheel walking. A comparison between both locomotion modes has been carried out in a simulation environment, providing information to decide the best locomotion mode from simplified terramechanic parameters such as the rolling resistance, slip ratio and slope. All of them could be derived from the rover proprioceptive sensors. The second novelty is related to the slip estimation during the execution of the wheel walking locomotion mode. In this sense, the paper presents a novel slip ratio estimator based on the acceleration of a wheel train. Its performance is analysed in the simulation environment, providing promising results.

The structure of this paper is as follows: Section 2 describes the interaction between the rover and terrain, modelled according to certain terramechanic parameters, the rover slip estimator during wheel walking is presented in Section 3. Finally, a simulation environment is used to perform a comparison between both locomotion modes and the performance of the slip ration estimator. The simulation environment is explained in Section 4 and obtained results are shown in Section 5. A set of conclusions and future works are explained in Section 7.

This paper performs a dynamic and kinematic analysis of two locomotion modes that would be used in reconfigurable rovers: normal driving and wheel walking. It is explained in Section 2, describing the interaction between the rover and terrain, modelled according to certain terramechanic parameters that can be derived from the rover proprioceptive sensors. In order to choose the best locomotion mode, the rover slip needs to be known, even during the execution of the wheel walking locomotion mode. So, Section 3 presents a novel slip ratio estimator based on the acceleration of a wheel train during the wheel-walking. A simulation environment is used to perform a comparison between both locomotion modes and the performance of the slip ratio estimator. The simulation environment is explained in Section 4 and the obtained results are shown in Section 5. Used materials, including an open source code repository are explained in Section 6. Finally, a set of conclusions and future works are explained in Section 7.

\section{Reconfigurable Rover-Terrain Interaction Model}

The interaction between rover wheels and terrain has been widely studied, providing complex equations to model the behaviour of the wheels during a rover traverse [20-22]. However, the aim of this paper is not going deeper on complex models, but using sensory measurements that would provide meaningful information about the wheel traction over a particular terrain. In this sense, several authors [23] simplify the wheel-terrain interaction using two parameters: rolling resistance $(\rho)$ and slip ratio $(\sigma)$. The rolling resistance represents the opposed force to the wheel motion, which is related to the wheel and terrain adhesiveness and friction. As explained in Reference [24], the rolling resistance is mainly caused by the hysteresis in the wheel used materials, friction between the wheel and the terrain caused by sliding. This parameter has been simplified to Equation (2) as proposed by several authors [25-27]. On the other hand, the wheel-soil interaction causes soil compression. Thus, the distance the wheel advances will be less than it is when free rolling. This effect is commonly called a slip. Therefore, the slip ratio has been defined as the relation between the ground truth and wheel speed $[13,24,28]$ which can be expressed as (1) It is commonly used to represent a second parameter that provides information about the wheel terramechanics.

$$
\begin{gathered}
\sigma=\frac{v_{d}-v_{r}}{v_{d}} \\
\rho=\frac{F_{r}}{F_{N}}
\end{gathered}
$$


In these equations, $v_{d}$ is the linear velocity of the wheels, $v_{r}$ is the ground-truth velocity of the rover body, $F_{r}$ is the rolling resistance contrary force and $F_{N}$ is the normal force, that is, the force the wheel exerts over the ground.

\subsection{Locomotion Modes}

During the traverse of a rover, the wheels' traction would be completely different if the soil is loose or compact. Depending on the terrain features, a reconfigurable rover would be able to adapt its locomotion mode to it, improving traction and power consumption. An example of a reconfigurable rover is the European Space Agency (ESA) Exomars 2020 rover [29], which has a $6 \times 6 \times 6$ drive configuration, composed of 6 wheels, 6 wheel walking/deployment joints and 6 steering joints. It allows the rover to perform several locomotion modes by the combination of the wheel walking joints and the wheels. This paper proposes the use of two locomotion modes whose dynamics are explained below.

\subsubsection{Normal Driving}

Normal driving represents the common locomotion mode for ground vehicles based on not legged wheels. Wheel walking joints remain locked and the drawbar pull is generated by the wheel motion. Figure 1 depicts this locomotion mode for the $i$ wheel of a rover equipped with $n$ wheels, and a slope $\alpha$ in the XZ plane. The wheel, with radius $r$, is located in $\left(x_{w i}, z_{w i}\right)$, while the walking joint, with length $l$, is located in $\left(x_{b i}, z_{b i}\right)$. A motor is attached to the wheel, which generates a torque $\tau_{w i}$ that induces an angular motion $\theta_{w i}$. Taking into consideration the slip (1), the drawbar pull force that is transmitted from the wheel can be calculated as:

$$
F_{d p w i}=(1-\sigma) \frac{\tau_{w i}}{r}
$$

On the other hand, the velocity of the wheel can be expressed as:

$$
\dot{x}_{w i}=\cos (\alpha) v_{r} \quad \dot{z}_{w i}=\sin (\alpha) v_{r}
$$

where the linear velocity of the wheels $v_{r}$ can be defined as:

$$
v_{r}=\dot{\theta}_{w i} r(1-\sigma)
$$

Therefore, according to D'Alembert principle [30], the linearised normal driving dynamics for the wheel $i$ can be represented as:

$$
\left(\frac{J_{w}}{r}+\frac{m_{b} r}{n}\right) \dot{v}_{d}+\rho \frac{m_{b}}{n} g \cos (\alpha) r+\frac{m_{b}}{n} g \sin (\alpha) r=\tau_{w i}(1-\sigma)
$$

Starting from the left, the first term is related to the wheel moment of inertia $J_{w}$ and the vehicle mass $m_{b}$, the second term is the rolling resistance (2) and the last term includes the vertical component of the gravity vector, parallel to the $Z$ axis. Slip $\sigma$ is considered an effect that reduces the transmitted torque $\tau_{w i}$ from the motor wheel. It is worth mentioning motor frictions are not considered.

Equation (6) provides information about the needed torque for each wheel to move the rover. Based on this torque $\tau_{w i}$, the required current can be calculated as:

$$
I_{N D}=\sum_{i=1}^{n} \int_{\Delta t} \frac{\tau_{w i} k_{w} \epsilon_{w}}{G_{w}}
$$

where, $n$ is the number of wheels, $k_{w}$ is the torque constant, that is., the relation between the exerted torque and the current consumption of the motor, $\epsilon_{w}$ is the gear-motor efficiency and $G_{w}$ is the gear ratio of the wheel motor. 


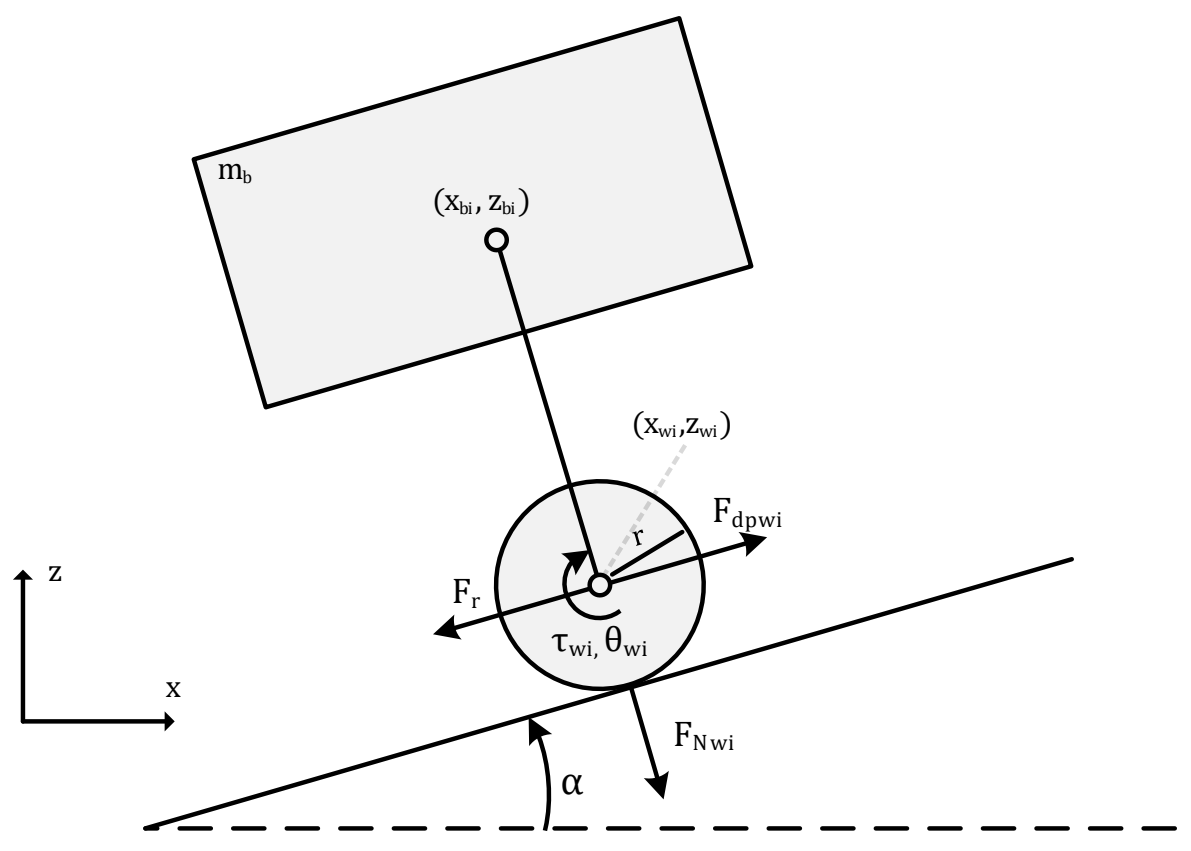

Figure 1. Diagram of the proposed normal driving model. Positions, forces and torques are depicted in order to illustrate the used notation and implied forces.

\subsubsection{Wheel Walking}

The wheel walking locomotion mode makes use of the motors located on the wheels and the wheel walking joints. The simplified model of a rover for the $\mathrm{XZ}$ plane is presented in Figure 2, illustrating how this mode works. A two wheels simplified model is considered, (w1) and (w2), each of them supported by a bar that is actuated by the wheel walking drive ( $b 1$ and $b 2)$. According to this configuration, interaction forces and torques are represented in the figure. Each wheel performs a movement that makes up the wheel walking mode-wheel-backward and wheel-forward. On one hand, the objective of the wheel-backward is to lock and move a wheel, $w 1$ in this case, backward in order to pull the rover body forward by a lever effect. On the other hand, the wheel-forward performs a contrary movement, which moves $w 2$ forward in order to balance the rover.

The wheel-backward movement performs a front to rear motion by applying a torque $\tau_{b 1}$ that moves the bar angle $\theta_{b 1}$ clockwise. This movement generates a drawbar pull force $F_{d p w 1}$ that is opposed to the friction force $F_{f}$ that depends on the normal force $F_{N w 1}$ in a similar way as stated in Equation (2). This friction is related to the terrain properties and if it is enough, it will avoid the wheel sliding backwards, pushing the rover forward. The dynamics of this movement can be represented using the D'Alembert principle as:

$$
J_{m} \ddot{\theta}_{b 1}+\frac{m_{b}}{n} \ddot{x}_{b 1} l \cos \left(\theta_{b 1}\right)+\frac{m_{b}}{n}\left(\ddot{z}_{b 1}+g\right) l \sin \left(\theta_{b 1}\right)=\tau_{b 1}
$$

where the first part represents the motor dynamics, defined by a moment of inertia $J_{m}$, motor frictions are ignored. The second part represents the body movement in the XZ axis, translated into torques. It is worth mentioning the wheel 1 is locked during the movement, that is, $\tau_{w 1}=\dot{\theta}_{w 1}=0 \mathrm{Nm}$. 
Therefore, there is no torque applied to this wheel during this locomotion mode. Based on this equation, the drawbar pull force on the wheel 1 can be expressed as:

$$
F_{d p w 1}=\frac{\cos \left(\theta_{b 1}\right)}{l} \tau_{b 1}
$$

and the drawbar pull force on the rover body is derived from:

$$
F_{d p b 1}=F_{d p w 1}-\left(F_{d p w 1}-F_{f}\right) ; F_{f} \leq F_{d p w 1}
$$

If we consider $\alpha=0$, kinematics of this movement can be represented as:

$$
x_{b 1}=l \sin \left(\theta_{b 1}\right)
$$

Therefore, to keep a fixed rover body linear velocity $\left(\dot{x}_{b 1}\right)$, the angular velocity of the walking joint $\left(\theta_{b 1}\right)$ can be calculated from the derivative of (11) as:

$$
\dot{\theta}_{b 1}=\frac{\dot{x}_{b 1}}{l \cos \left(\theta_{b 1}\right)}
$$

On the other hand, the wheel-forward movement performs the contrary motion, synchronised with the wheel-backward one, in order to keep fixed the rover linear velocity. The bar is moved forward to place the wheel at the front side. For this purpose, a torque $\tau_{b 2}$ is generated to move the bar forward, and at the same time, the wheel is also moved by generating a torque $\tau_{w 2}$. Both torques generates a drawbar pull force $F_{d p w 2}$ on the wheel whose dynamics can be represented as:

$$
J_{w} \ddot{\theta}_{w 2}+m_{w} \dot{v}_{d} r+\rho F_{N w 2} r+\frac{m_{b}}{n} l \sin \left(\theta_{b 2}\right)\left(\ddot{z}_{b}+g \cos (\alpha)\right)=\tau_{w 2}(1-s)+\tau_{b 2} \cos \left(\theta_{b 2}\right) \frac{r}{l}=F_{d p w 2} r
$$

where the first term represents the rotational inertia $J_{w}$ of the wheel, the second one represents the linear inertia of the wheel mass $m_{w}, \rho F_{N w 2}$ represents the rolling resistance (2), and the fourth term represents the vertical forces due to the gravity $g$ and the rover body vertical inertia. On the other hand, the torque $\tau_{w 2}$ is generated by the wheel motor and it is affected by the slip coefficient, and the generated torque $\tau_{b 2}$ is translated to its corresponding torque in the wheel as stated in the equation.

Taking into consideration $\alpha=0$ again, kinematics of this movement can be defined as:

$$
x_{b 2}=x_{w 2}+l \sin \left(\theta_{b 2}\right)
$$

whose derivative is:

$$
\dot{x}_{b 2}=\dot{x}_{w 2}+l \dot{\theta}_{b 2} \cos \left(\theta_{b 2}\right)
$$

Assuming $\dot{x}_{b 1}=\dot{x}_{b 2}$, and therefore, $\dot{\theta}_{b 2}=-\dot{\theta}_{b 1}$, which keep the rover going straight forward, the angular velocity of the wheel $\dot{\theta}_{w 2}$ is calculated as:

$$
\dot{\theta}_{w 2}=\frac{2 \dot{x}_{b 2}}{r}
$$

Finally, the current consumption for a step using this locomotion mode can be appreciated in (17). In this equation, $\tau_{w i}$ and $\tau_{b i}$ are the torque from each wheel walking joint and wheel respectively, $k_{w}$ and $k_{b}$ are the motor torque constants, $\epsilon$ is the motor efficiency, and $G$ is the ratio of the motor gear. It is worth mentioning for a multiple wheels rover, wheels 1 to $N / 2$ perform the wheel-backward movement and wheels $N / 2+1$ to $N$ the wheel-forward.

$$
I_{W W}=\sum_{i=1}^{N / 2} \int_{\Delta t} \frac{\left|\tau_{b i}\right| k_{T} \epsilon_{b i}}{G_{b i}}+\sum_{i=N / 2+1}^{N} \int_{\Delta t} \frac{\left|\tau_{w i}\right| k_{T} \epsilon_{w i}}{G_{w i}}+\sum_{i=N / 2+1}^{N} \int_{\Delta t} \frac{\left|\tau_{b i}\right| k_{T} \epsilon_{b i}}{G_{b i}}
$$




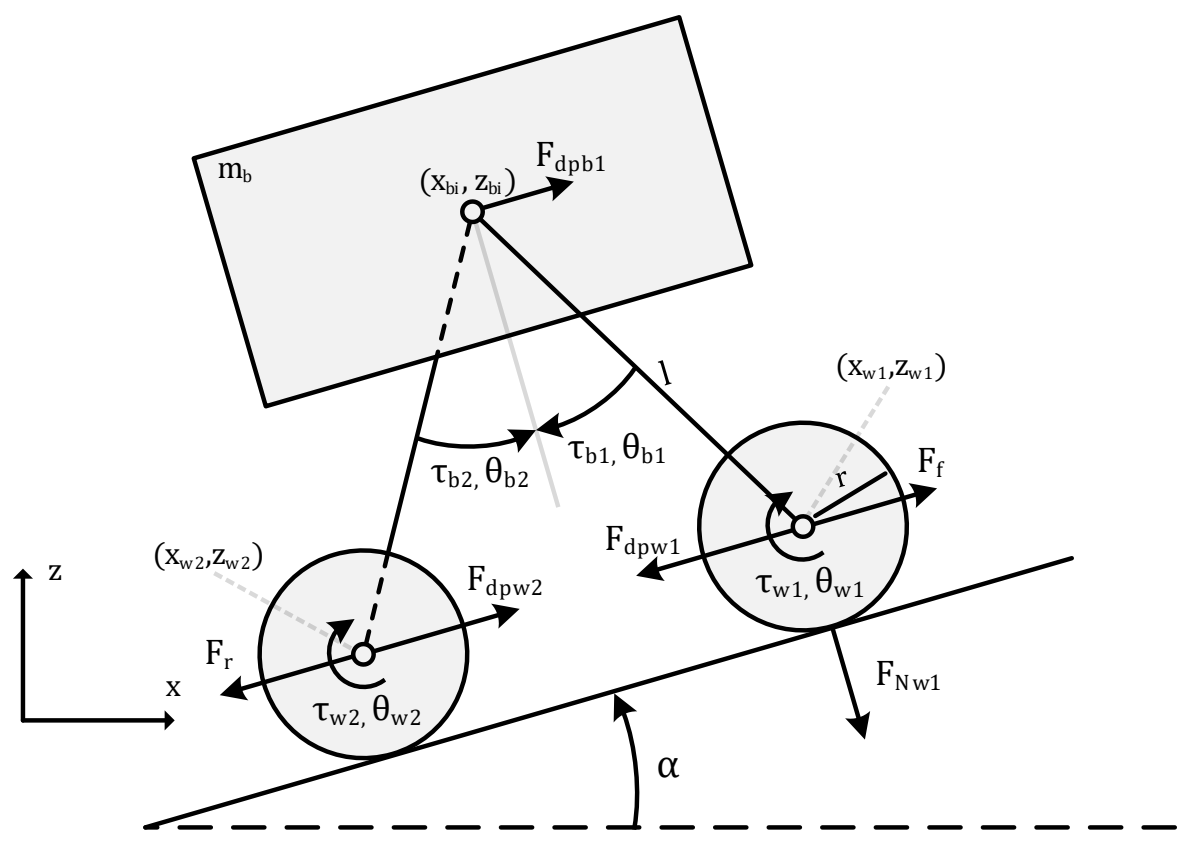

Figure 2. Diagram of the proposed wheel walking mode. It represents both steps, wheel-backward and wheel-forward that are carried out by wheels $w 1$ and $w 2$, respectively.

\section{Slip Estimation in Wheel Walking}

Having defined both locomotion modes and its dynamics, a method for estimating online the best one in terms of performance would be needed. By means of the terrain features, a comparison between both locomotion modes could be carried out, and the relationship between these features and the performance of each one would help to decide the best locomotion mode.

Based on Equations (6), (8) and (13), parameters that define the terrain features and affect the rover dynamics are the slip ratio (1) and the rolling resistance (2). In the case of normal driving, there are many methods for estimating these parameters. In planetary exploration, which implies the absence of a GPS signal, the slip ratio can be calculated by the difference between the wheel odometry and the ground truth velocity that can be estimated by means of Visual Odometry [31]. Other authors propose the use of the lug traces to estimate the slip ratio [32]. On the other hand, the rolling resistance can be estimated using different methods that span from specific sensors, as acoustic [33] or optical [34] ones, to the use of artificial intelligence, for example, neural networks [35].

As stated, the estimation of these parameters is crucial for deciding the best locomotion mode every instant, which would make the rover switch from one to another. Although it would be easy to estimate these parameters in normal driving, they remain to be investigated during the execution of the wheel walking mode. The main difficulty is finding a method to estimate the rolling slip when the wheels are moving forward and backward. It can be done easily in normal driving, as stated before. However, this method is not valid for wheel walking, because the wheels are moved in a different way, causing different wheel-terrain interaction compared to normal driving. Therefore, the challenge is to estimate the slip ratio as it were carried out in normal driving, which would be useful to determine the instant time to switch from wheel walking to normal driving. This section introduces a novel method to estimate the slip ratio for a rover when it is executing the wheel walking mode.

During the wheel walking locomotion mode, the slip mainly affects the wheel-forward movement, meanwhile the wheel-backward movement is affected by friction forces because the wheel is not rolling 
itself. So, the normal driving slip ratio could be estimated by analysing the wheel motion during the wheel-forward movement. For this purpose, an acceleration step has been added. This step would be executed sometimes during the wheel walking. It consists of increasing the speed of the wheels that are carrying out the wheel-forward motion. During this step, the relation between the real and ideal distance covered by the wheels is analysed. If both distances are similar the slip ratio is low, and if the real distance is significantly lower than the ideal one, it means the slip ratio is high.

Figure 3 represents the distance $\left(d_{w}\right)$ a wheel covers in a wheel-forward movement. Assuming the movement starts at $t=0$ and finishes at $t=t_{w f}, \theta_{b 2}(0)$ and $\theta_{b 2}\left(t_{w f}\right)$ represent the initial and final angular position of the walking joints respectively; and $\theta_{w 2}(0)$ and $\theta_{w 2}\left(t_{w f}\right)$ represent the initial and final angular position of the wheels. As regards the position of the wheels and the rover base, $\left(x_{b 2}(0), z_{b 2}(0)\right)$ and $\left(x_{b 2}\left(t_{w f}\right), z_{b 2}\left(t_{b f}\right)\right)$ represent the initial and final position of the wheel walking joint, and $\left(x_{w 2}(0), z_{w 2}(0)\right)$ and $\left(x_{w 2}\left(t_{w f}\right), z_{w 2}\left(t_{w f}\right)\right)$ represent the initial and final position of the wheel that is carrying out the wheel-forward movement.

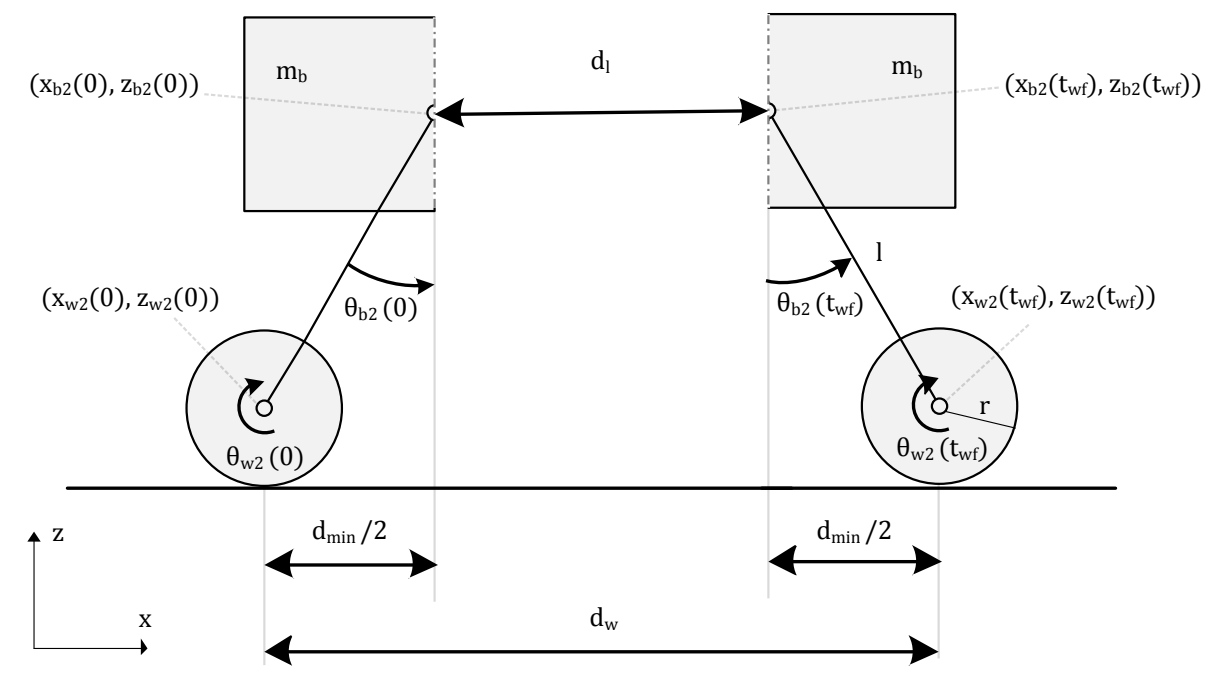

Figure 3. Distance covered by the wheels during the acceleration step. It is used to estimate the slip ratio.

During the acceleration step, the rolling motion of the wheels pushes the rover a distance $\left(d_{l}\right)$ that is proportional to the increment of the wheel speed with respect to the default speed that it has due to the wheel-forward movement itself $\left(d_{\mathrm{min}}\right)$. When the slip ratio is high, i.e., close to $1, d_{l}$ is negligible during the acceleration step. So, the distance covered by the wheel approaches the minimum distance $\left(d_{w} \approx d_{\min }\right)$. The minimum distance can be calculated as:

$$
d_{\min }=l \sin \left(\theta_{b 2}(0)\right)+l \sin \left(\theta_{b 2}\left(t_{w f}\right)\right)
$$

The opposite situation arises when the slip ratio is very low (around 0 ). The distance covered by the wheels is the maximum distance $\left(d_{\max }\right)$, which can be calculated as stated in (19).

$$
d_{\max }=d_{\min }+d_{l}=d_{\min }+\left(\theta_{w 2}\left(t_{w f}\right)-\theta_{w 2}(0)\right) r
$$


By a linear regression with this two distances and their corresponding slip ratios, we obtain the Equation (20). It provides the real slip ratio $(\sigma)$ in function of the distance covered by a certain wheel in a single forward walking step $\left(d_{w}\right)$.

$$
\sigma=\frac{d_{\max }-d_{w}}{d_{\max }-d_{\min }}
$$

As can be appreciated in (20), small intervals between $d_{\max }$ and $d_{\min }$ decreases the accuracy of the slip ratio estimation: the possible values for $d_{w}$ would be only a few. As a consequence, a low wheel velocity would lead into low slip ratio accuracy. So, for vehicles that move slowly like rovers, it is necessary to increase the wheels speed.

However, the acceleration step increases the lineal velocity of the wheel-forward train of the rover, which induces the rover to perform a curve. Figure 4 represents the accelerated step for a wheel-forward motion that is carried out with the right wheel train. Assuming the behaviour of the rover is similar to a skid-steering rover during the acceleration step, an instant centre of rotation (ICR) can be defined [36], whose turning radius $R$ can be calculated as:

$$
R=\frac{\dot{x}_{b 2}+\dot{x}_{b 1}}{\dot{x}_{b 1}-\dot{x}_{b 2}} d_{c}
$$

where $d_{c}$ is the distance from the wheel to the centre of the rover, $\dot{x}_{b 1}$ can be obtained from (12) and $\dot{x}_{b 2}$ is affected by the wheel speed increment $\dot{\theta}_{a}$ as:

$$
\dot{x}_{b 2}=\left(\dot{\theta}_{w 2}+\dot{\theta}_{a}(1-\sigma)\right) r+l \dot{\theta}_{b 2} \cos \left(\theta_{b 2}\right)
$$

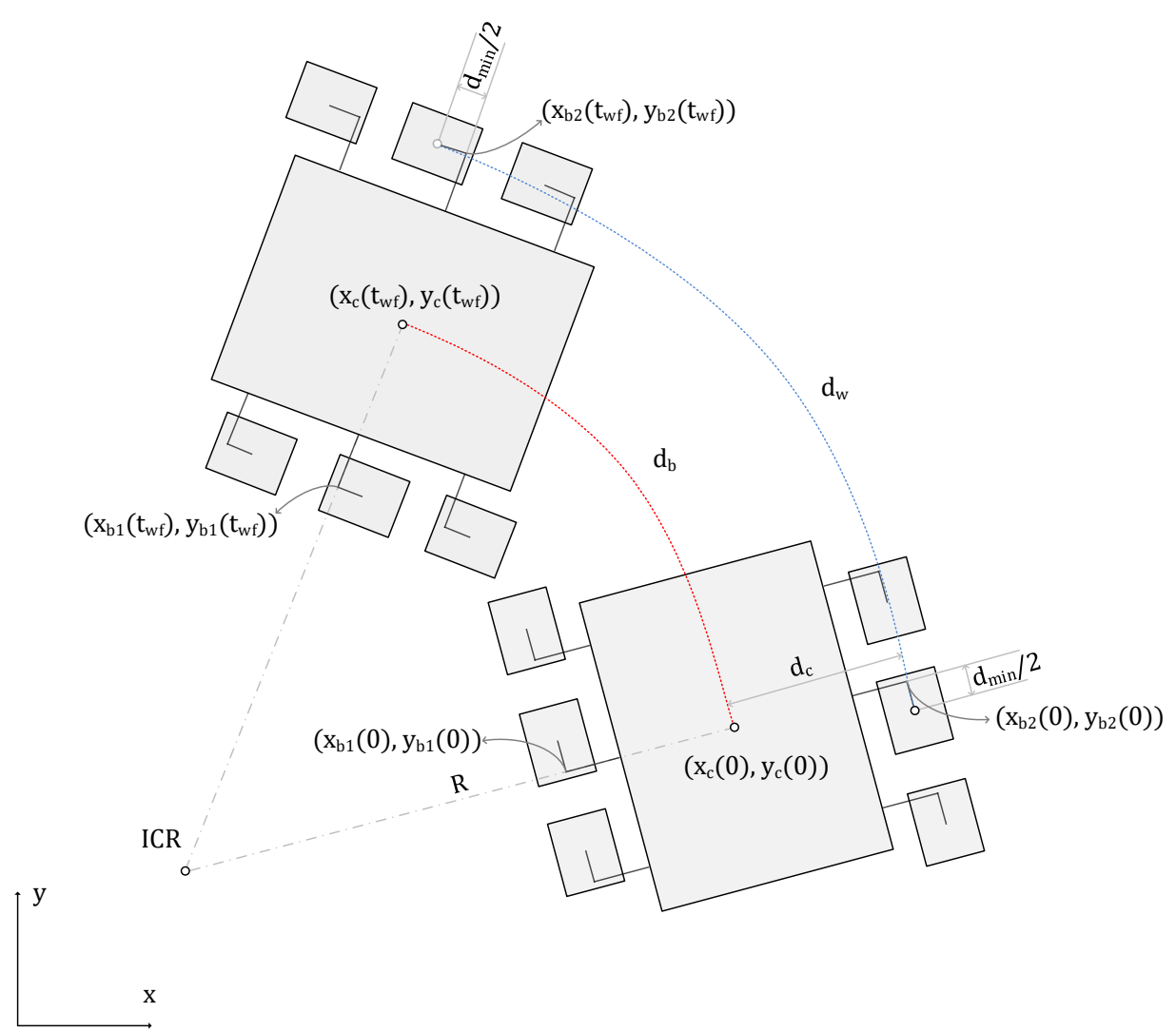

Figure 4. Diagram that shows the trajectory of the rover in the XY plane for the acceleration step. As shown, the rover performs a curve, whose turning radius can be estimated as an ICR with a radius $R$. 


\section{Simulation Environment}

A comparison between both locomotion modes and a validation of the slip estimation method has been carried out by means of a simulation environment. Its architecture is shown in Figure 5 . The rover was design in SolidWorks and its motor parameters were taken from the real rover. The 3D model of the rover and its dynamics were exported to the Vortex Studio Simulation Environment. This platform includes an advanced multibody physics engine that is able to provide advanced 3D graphics and integration with other platforms as Matlab-Simulink. Indeed, Vortex Studio has been previously used to perform simulations of rovers on soft terrains [37].

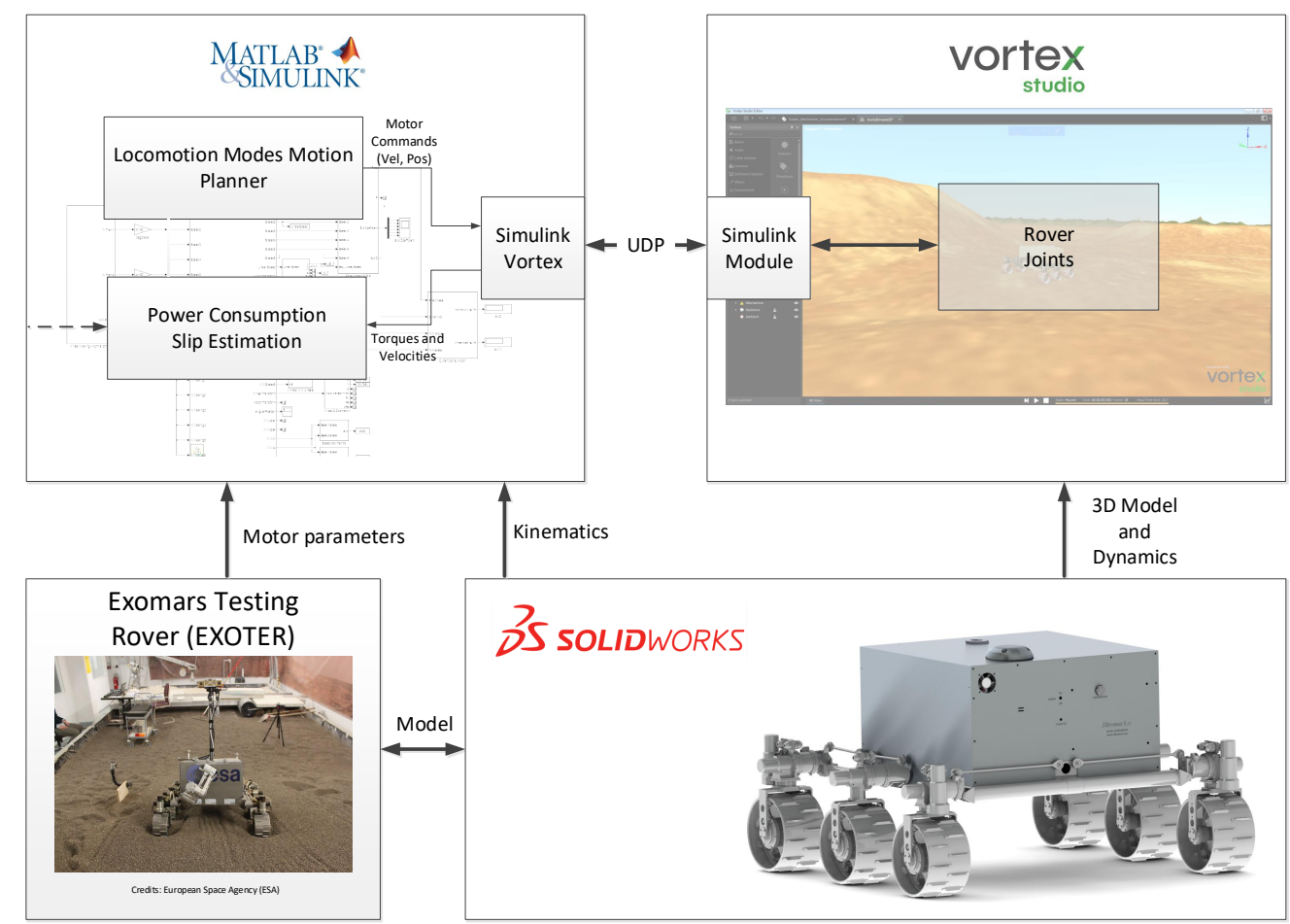

Figure 5. Simulation environment. It shows interactions between the used software. The 3D model of the ExoTeR rover is obtained from SolidWorks and included within Vortex Studio. Matlab \& Simulink is used to perform the locomotion modes motion planner, retrieve power consumption and estimate the slip ratio during wheel walking.

On the other hand, the rover kinematics was used to develop a motion planner in Matlab-Simulink. It generates the rover motor commands each instant time according to the chosen locomotion mode. Commands were sent to Vortex Studio through an UDP communication interface, and the rover joint torques and velocities were communicated from Vortex Studio to Matlab-Simulink through the same interface. This information was used to calculate the power consumption in real-time using the real rover motor parameters. Moreover, the slip ratio was estimated during the execution of the wheel walking mode, as proposed in Section 3.

As regards the rover kinematics and dynamics, Table 1 includes all the needed parameters that were used in previous sections. They were included in the simulation environment. 
Table 1. Rover ExoTeR dynamic parameters.

\begin{tabular}{cccc}
\hline Parameter & Symbol & Value & Unit \\
\hline Number of wheels & $N$ & 6 & \\
Wheel radius & $r$ & 0.07 & $\mathrm{~m}$ \\
Wheel-boogie bar length & $l$ & 0.125 & $\mathrm{~m}$ \\
Gravity & $g$ & 9.8 & $\mathrm{~m} / \mathrm{s}^{2}$ \\
Wheel mass & $m_{w}$ & 0.484 & $\mathrm{~kg}$ \\
Rover body mass & $m_{b}$ & 11.4 & $\mathrm{~kg}$ \\
Wheel inertia & $J_{w}$ & $9.8 \times 10^{-4}$ & $\mathrm{kgm}^{2}$ \\
\hline
\end{tabular}

The motor parameters that are used in the ExoTeR rover are shown in Table 2. These parameters are used to compute the total rover power consumption during the execution of the locomotion modes. The motor used is the Maxon DC motor (Part. number: 110160) that moves the wheel and walking joints. The wheel motor is provided with a gear that increases the motor torque despite a reduced efficiency. On the other hand, the walking joint motor is provided with another gear, and also connected to a mechanical Remote Centre of Motion (RCM) that provides, both together, a high gear ratio. The objective of the RCM is to allow the boogie rotate at the same time the wheels, keeping them perpendicular to the ground.

Table 2. Rover ExoTeR motor parameters.

\begin{tabular}{cccccccc}
\hline \multicolumn{3}{c}{ Wheel Drive } & \multicolumn{7}{c}{ Walking Joint Drive } \\
\hline & \multicolumn{7}{c}{ Model: Maxon DC Motor A-Max No.110160 } \\
\hline Parameter & Symbol & Value & Unit & Parameter & Symbol & Value & Unit \\
\hline Gear ratio & $G_{w}$ & 675.545 & - & Gear ratio & $G_{b}$ & 1900 & - \\
Efficiency ratio & $\epsilon_{w}$ & 0.6 & - & Efficiency ratio & $\epsilon_{b}$ & 0.49 & - \\
Torque constant & $k_{w}$ & 10.9 & $\mathrm{mNm} / \mathrm{A}$ & Torque constant & $k_{b}$ & 10.9 & $\mathrm{mNm} / \mathrm{A}$ \\
Rotor inertia & $J_{m}$ & 4.36 & $\mathrm{gcm}^{2}$ & Rotor inertia & $J_{m}$ & 4.36 & $\mathrm{gcm}^{2}$ \\
\hline
\end{tabular}

Finally, to analyse the rover-terrain interaction and both locomotion modes, six terrains have been defined. They have been configured taking into consideration the flat slip ratio $(\sigma)$ and rolling resistance $(\rho)$ from low to high values. Based on the Vortex Studio interaction model, five parameters have been modified to configure them. Table 3 shows the parameter values for each terrain. The two first parameters, stiffness and damping, are related to the wheel sinkage during the movement, and it affects the wheel contact points as shown in Figure 6. It is directly related to the wheel adherence to the terrain. On the other hand, slip, friction coefficient and the static friction scale represent the wheel-terrain interaction when it is rolling. The slip parameter is related to the inverse of the viscous friction, which affects the wheel slip, the friction coefficient is defined as the contrary force that is exerted due to the rolling resistance as stated in (2). At last, the static friction scale defines the force to be carried out to move the wheel when it is stopped. The limit in the contact friction force is multiplied by this scale.

Table 3. Terrain parameters.

\begin{tabular}{ccccccc}
\hline Terrain & $\mathbf{1}$ & $\mathbf{2}$ & $\mathbf{3}$ & $\mathbf{4}$ & $\mathbf{5}$ & $\mathbf{6}$ \\
\hline Stiffness $(\mathrm{N} / \mathrm{m})$ & 2000 & 300 & 250 & 150 & 75 & 50 \\
Damping $(\mathrm{kg} / \mathrm{s})$ & 20,000 & 850 & 800 & 400 & 350 & 200 \\
Slip (s/kg) & 0.0005 & 0.01 & 0.015 & 0.02 & 0.03 & 0.03 \\
Friction coefficient & 0.9 & 0.8 & 0.5 & 0.4 & 0.3 & 0.1 \\
Static friction scale & 1.2 & 1.2 & 1.2 & 1.2 & 1.2 & 1.2 \\
\hline
\end{tabular}




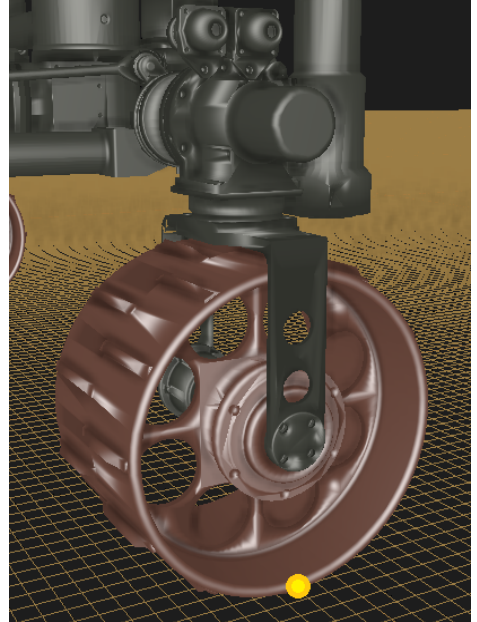

(a) Terrains 1 and 2 .

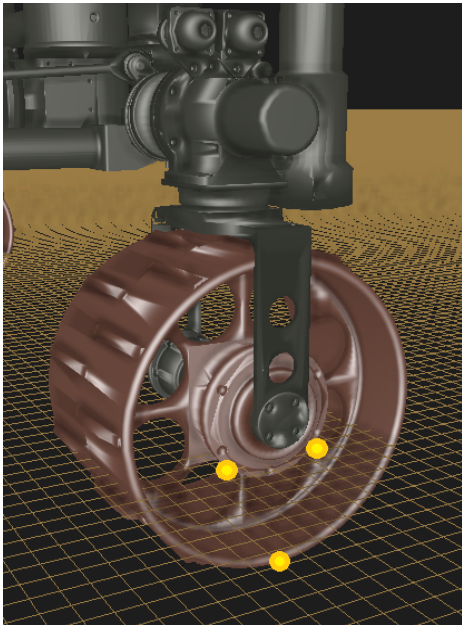

(b) Terrains 3 and 4 .

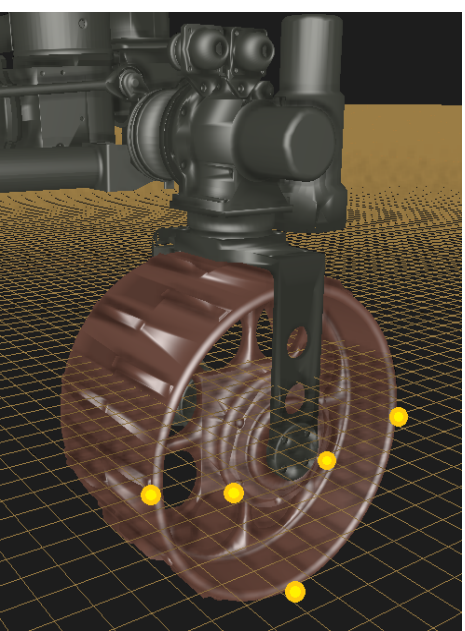

(c) Terrains 5 and 6.

Figure 6. Vortex Studio defined contact points for different terrains. Depending on the Stiffness and Damping parameters, these contact points can be increased or decreased. (a) Terrains 1 and 2; (b) Terrains 3 and 4; (c) Terrains 5 and 6.

These terrains were implemented in the simulation environment and the rover-terrain interaction was analysed in order to obtain the slip ratio $\sigma(1)$ and rolling resistance $\rho$ (2) in a flat terrain, i.e., $\alpha=0$. Table 4 shows the obtained parameters for the six terrains.

Table 4. Equivalent parameters of the six terrains.

\begin{tabular}{ccccccc}
\hline Terrain & $\mathbf{1}$ & $\mathbf{2}$ & $\mathbf{3}$ & $\mathbf{4}$ & $\mathbf{5}$ & $\mathbf{6}$ \\
\hline Flat Slip Ratio $(\sigma)$ & 0 & 0.0733 & 0.1985 & 0.4764 & 0.65 & 0.95 \\
Rolling Resistance Coefficient $(\rho)$ & 0.0036 & 0.0663 & 0.1180 & 0.2142 & 0.2474 & 0.2423 \\
\hline
\end{tabular}

\section{Wheel Walking Motion Planning}

To perform the wheel walking mode, motion of the rover joints and wheels needs to be planned in order to synchronise them and make the rover go ahead keeping a fixed linear velocity. To this end, Equations (12), (15) and (16) are used to calculate joint velocities taking into consideration a fixed rover linear velocity. Figure 7 represents the planned velocity profile for a walking joint and wheel (orange) and the real velocity of the joint and wheel during the execution of a simulation (blue). The profile is repeated by the rest of the wheels that are at the same side, meanwhile, the wheels on the other side perform the contrary step, e.g., the right side wheel-forward and the left side wheel-backward. As shown, the rover is able to follow the references without excessive error.

The defined profile generates a rover linear speed that is represented in Figure 8. In this figure, the ideal rover speed is represented with an orange line, and the real speed, obtained from the simulation environment, is represented in blue. The rover speed was fixed to $0.08 \mathrm{~m} / \mathrm{s}$, a suitable speed for rovers [38]. However, it is not constant because of the wheel walking joint controller that is implemented in the simulation environment. As shown in Figure 7, a change in the joint velocity causes a peak that is transmitted to the linear velocity of the rover. 


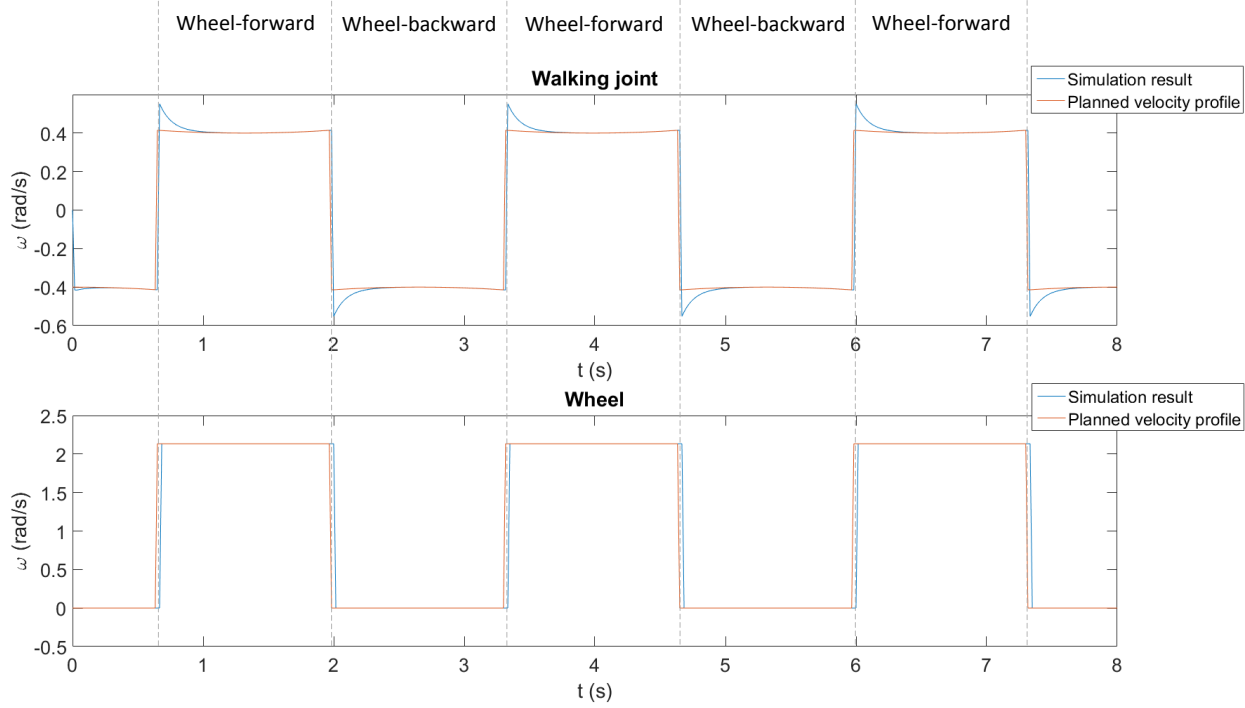

Figure 7. Ideal and real angular velocities of the walking joints and wheels. As shown, the walking joint generates a peak for each step. It is due to the joint controller implemented in Vortex Studio. The wheel is able to follow the velocity reference with a small time delay.

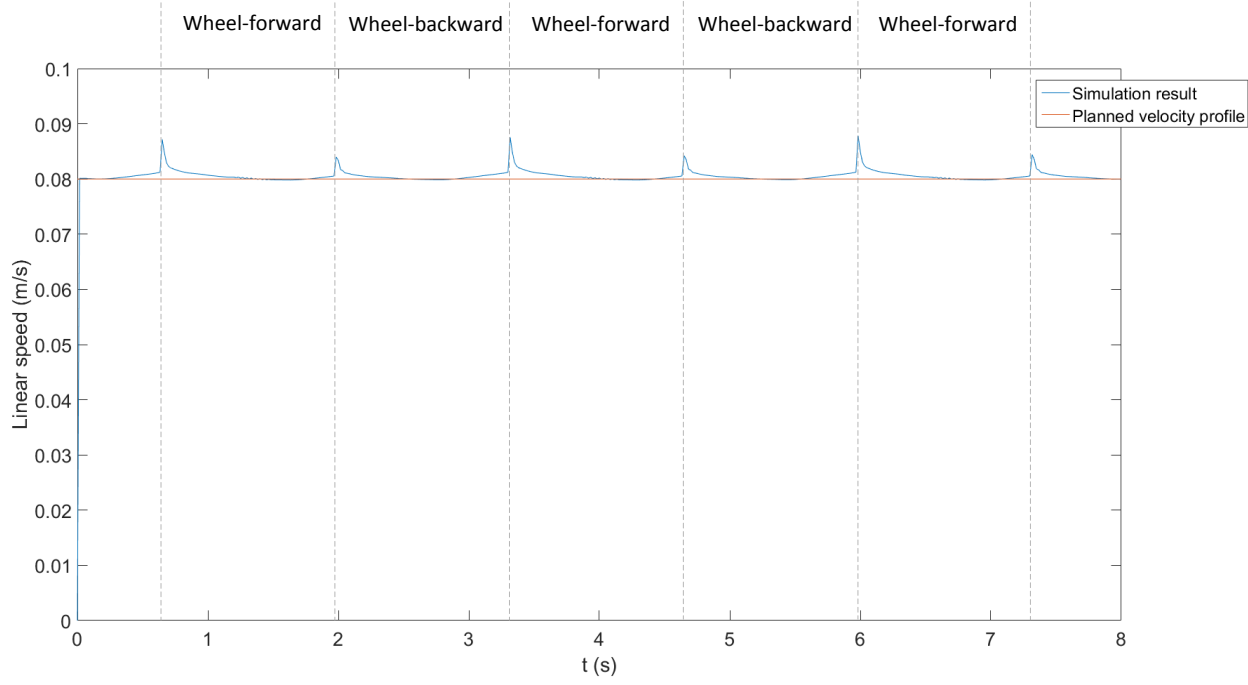

Figure 8. Rover linear velocity during wheel walking. Peaks on the walking joints causes the same effect over the linear velocity of the rover.

\section{Results}

Once the simulation environment and the wheel walking motion planner have been defined, several simulations have been carried out for two purposes. The first one is to perform a comparison between both locomotion modes, providing the best one depending on the six previously defined terrains. The second simulation is related to the analysis of the slip ratio estimation during the wheel walking, as stated in Section 3. As previously mentioned, it is carried out by increasing the wheel velocity during the wheel forward step. An analysis of the wheel velocity and the estimation accuracy is carried out in this section.

\subsection{Normal Driving vs Wheel Walking}

To perform a comparative between both locomotion modes, they were executed for each terrain and slope five times. The simulation time was $17 \mathrm{~s}$ for each one. Average of obtained measurements was 
represented in the following figures, which helped to decide the best locomotion mode depending on the terrains and its corresponding parameters. Moreover, a video (https://youtu.be/h6_WXRw_4-o) was recorded in order to illustrate the performed simulations.

Figure 9 shows the current consumption for both locomotion modes on different terrains and slopes. As shown, the current consumption gets increased as the slope does. However, the difference between both locomotion modes gets decreased as the slip and friction coefficient are increased, i.e., from terrain 1 to 6 . The wheel walking mode is mainly affected by the rolling resistance, an increase of it causes higher current consumption by the loss of inertia when the rover changes from one step to another. In the case of normal driving, the current consumption is also increased because the rolling resistance counteracts the drawbar pull force. It is worth mentioning that the rolling resistance is related to the wheel contact points that are considered in the simulation environment. Each contact point offers a rolling resistance, so the whole wheel consumption is higher in function of how many contact points are considered, which depends on the wheel sinkage.

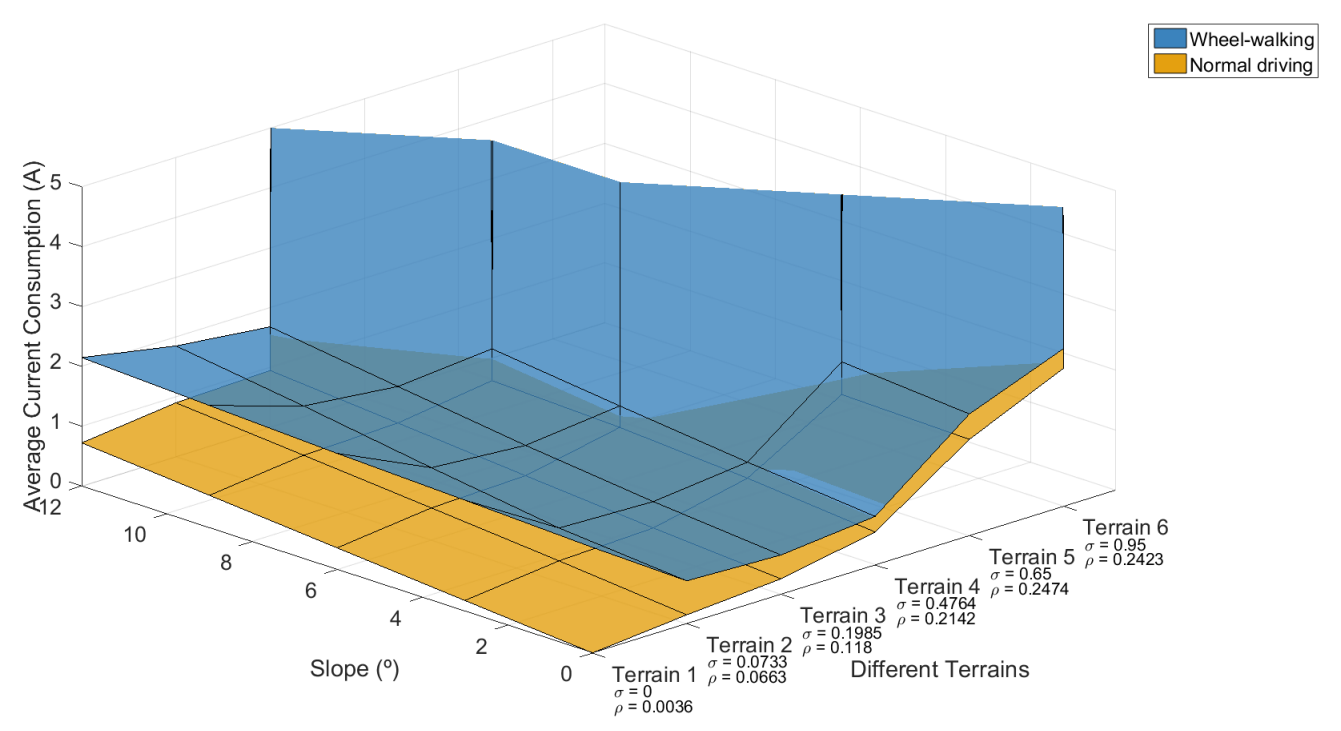

Figure 9. Average current consumption of wheel walking and normal driving locomotion modes on different terrains. It is always lower in normal driving, however, the difference is decreased as $\sigma$ and $\rho$ are increased.

As a conclusion, normal driving always consumes less current than wheel walking, but it is not better on every terrain, so comparing the average speed between the two locomotion modes is also needed, to decide the best one for each case. Figure 10 shows the average speed of both locomotion modes for different terrains. When the slip ratio is lower than 0.2 (terrains 1 and 2), normal driving is faster than wheel walking. Conversely, rover speed decreases drastically when slip ratio gets increased for normal driving, but the speed is softly decreased in wheel walking, keeping the wheel walking speed higher. High slope and slip ratio cause the rover to stop or even move backward. This effect is represented in the figure as zero speed. It can be appreciated with terrains 5 and 6 and a slope higher than 2 degrees. 


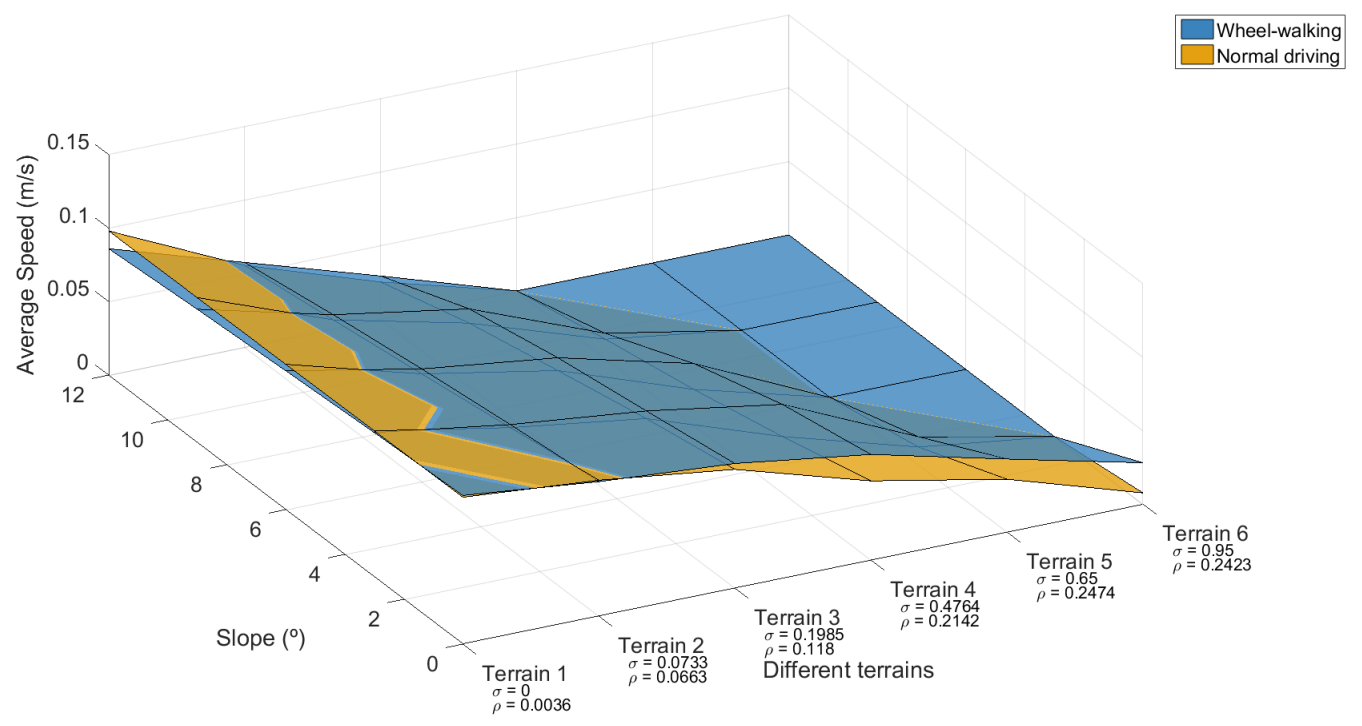

Figure 10. Average speed of wheel walking and normal driving locomotion modes on different terrains. normal driving velocity is higher than wheel walking only for very low $\sigma$ and $\rho$.

Once both locomotion modes have been analysed in terms of current consumption and speed, the relation between these parameters can be used to provide the best locomotion mode for each terrain with different properties. For this purpose, Figure 11 represents the average energy consumption per distance for each terrain and different slopes.

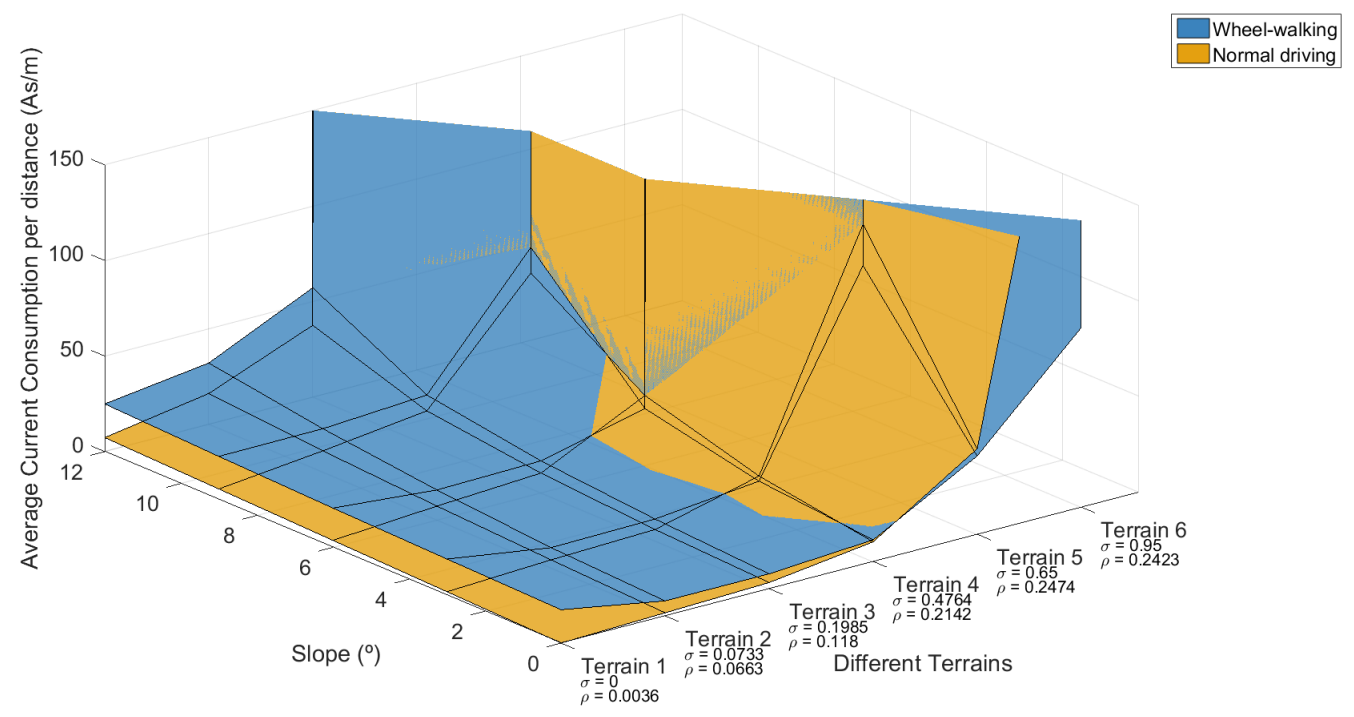

Figure 11. Relation between current consumption and speed of both locomotion modes, in function of slope and for different terrains. As shown, normal driving provides lower As/m than wheel walking for terrains 1 to 3 . wheel walking shows a better performance in terrains 4 to 6 and slopes lower than $7^{\circ}$.

Based in this information, Equation (23) has been defined to provide a score that indicates the best locomotion mode (BLM).

$$
B L M=\left(A V_{W W}-A V_{N D}\right) \in[-30,30]\left(\frac{A s}{m}\right)
$$


It provides the difference between both locomotion modes current/speed data, where $A V_{N D}$ is a matrix with the data of normal driving represented in Figure 11, and $A V_{W W}$ is a similar matrix with the data of wheel walking. Positive values of $B L M$ represent when normal driving is the best locomotion mode to use, and negative values do the same with wheel walking. Figure 12 represents the obtained matrix where yellow corresponds to normal driving, blue is wheel walking and green is neutral. Non plotted areas (grey in the figure) represent a situation in which the rover loses traction and therefore it would stop or move backwards.

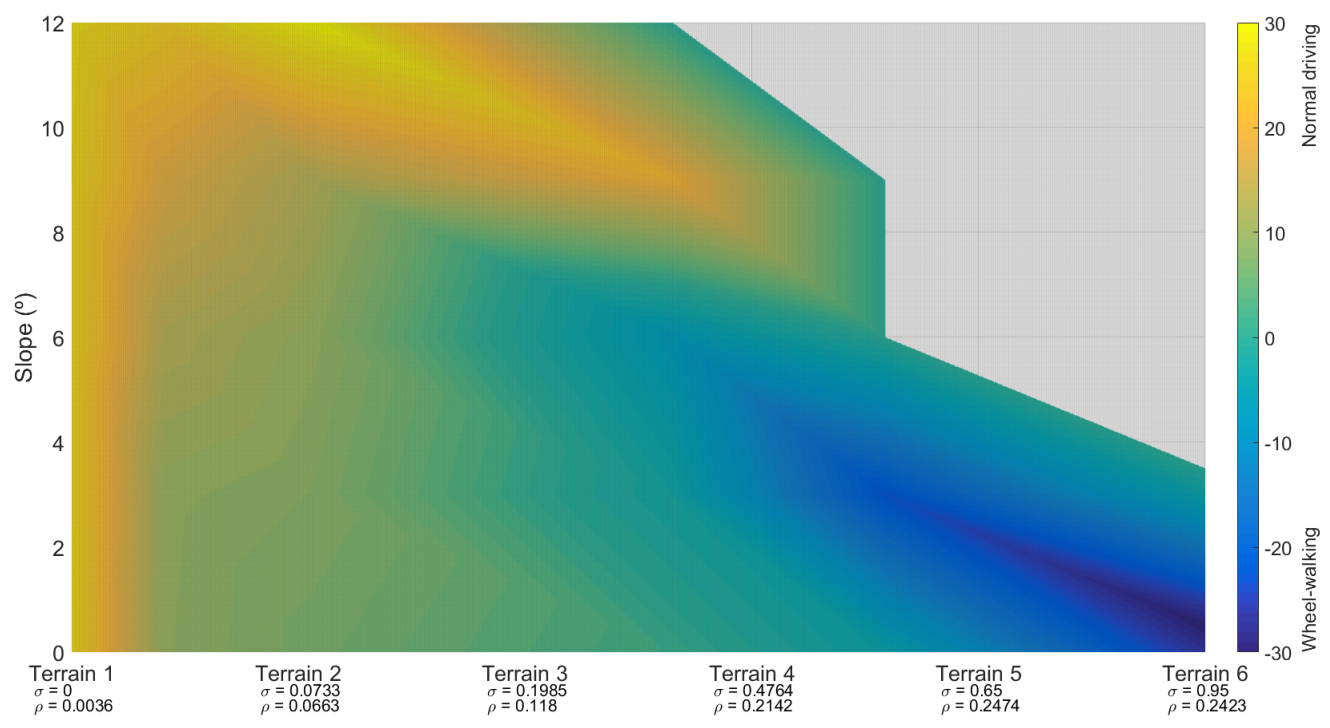

Figure 12. Best locomotion mode in function of slope and terrain. This figure shows how normal driving is the best one in most of cases, and wheel walking is suitable for terrains with $\sigma \geq 0.47$, $\rho \geq 0.21$. It is worth mentioning that grey area in the figure represents terrains and slopes that cannot be overcome by the rover due to the terrain parameters and the slope.

It is important to clarify the best locomotion mode depends on the rover dynamics. Previous figures are related to the particular ExoTeR rover. However, it is clear the best locomotion mode depends mostly on the terrain features and slightly on the slope. As shown in Figure 12, for the particular case of the ExoTeR rover, normal driving would be recommended for terrains 1 to $3(\sigma \leq 0.1985$ and $\rho \leq 0.118)$. In the case of terrain $4(\sigma \simeq 0.1985$ and $\rho \simeq 0.118)$, the use of wheel walking would be recommended for low slopes $\left(\leq 6^{\circ}\right)$ and normal driving for slopes higher than $6^{\circ}$.

\subsection{Slip Ratio Estimation}

Section 3 describes a method for obtaining the terrain slip ratio by increasing the wheel speed during the wheel-forward step in wheel walking. To validate this method, the previously described terrains (Table 4) were used to perform some simulations with different speed increments. In particular, a $20.6 \mathrm{~s}$ simulation was carried out for each terrain and speed increment. The results are shown in Table 5. As can be appreciated, depending on the terrain and the speed increment, the slip estimation varies with respect to the real one. 
Table 5. Obtained slip ratios for the different terrains and wheel velocities.

\begin{tabular}{ccccccc}
\hline Terrain & $\mathbf{1}$ & $\mathbf{2}$ & $\mathbf{3}$ & $\mathbf{4}$ & $\mathbf{5}$ & $\mathbf{6}$ \\
\hline Real slip ratio $(\sigma)$ & 0 & 0.0734 & 0.1907 & 0.4764 & 0.645 & 0.95 \\
Vel x1 & 0.2644 & 0.3440 & 0.4058 & 0.5058 & 0.5908 & 0.8907 \\
Vel x1.25 & 0.1975 & 0.3068 & 0.3669 & 0.4602 & 0.6213 & 0.9283 \\
Vel x1.5 & 0.1478 & 0.2582 & 0.3229 & 0.4307 & 0.6472 & 0.9359 \\
Vel x1.75 & 0.1137 & 0.2094 & 0.2706 & 0.3890 & 0.6775 & 0.9349 \\
Vel x2 & 0.0874 & 0.1689 & 0.2316 & 0.4003 & 0.7156 & 0.9268 \\
Vel x3 & 0.01 & 0.1133 & 0.2149 & 0.5607 & 0.8129 & 0.9172 \\
Vel x4 & 0 & 0.0876 & 0.2197 & 0.6612 & 0.8600 & 0.9235 \\
Vel x5 & 0 & 0.0778 & 0.2295 & 0.7210 & 0.8895 & 0.9403 \\
\hline
\end{tabular}

Taking into account this information, Figure 13 was generated. It shows a comparison between different speed increments and the maximum slip estimation error for each terrain. It can be clearly appreciated the estimation errors for low speed increments ( $x 1$ and $x 1.4)$ are higher for terrains 1 to 3 and lower for terrains 4 to 6 . On the contrary, the estimation errors for high speed increments (x3, $\mathrm{x} 4$ and $\mathrm{x} 5$ ) are higher for terrains 4 to 6 and lower for terrains 1 to 3 . Therefore, a speed increment $\mathrm{x} 2$ arises as the best choice for all the terrains.

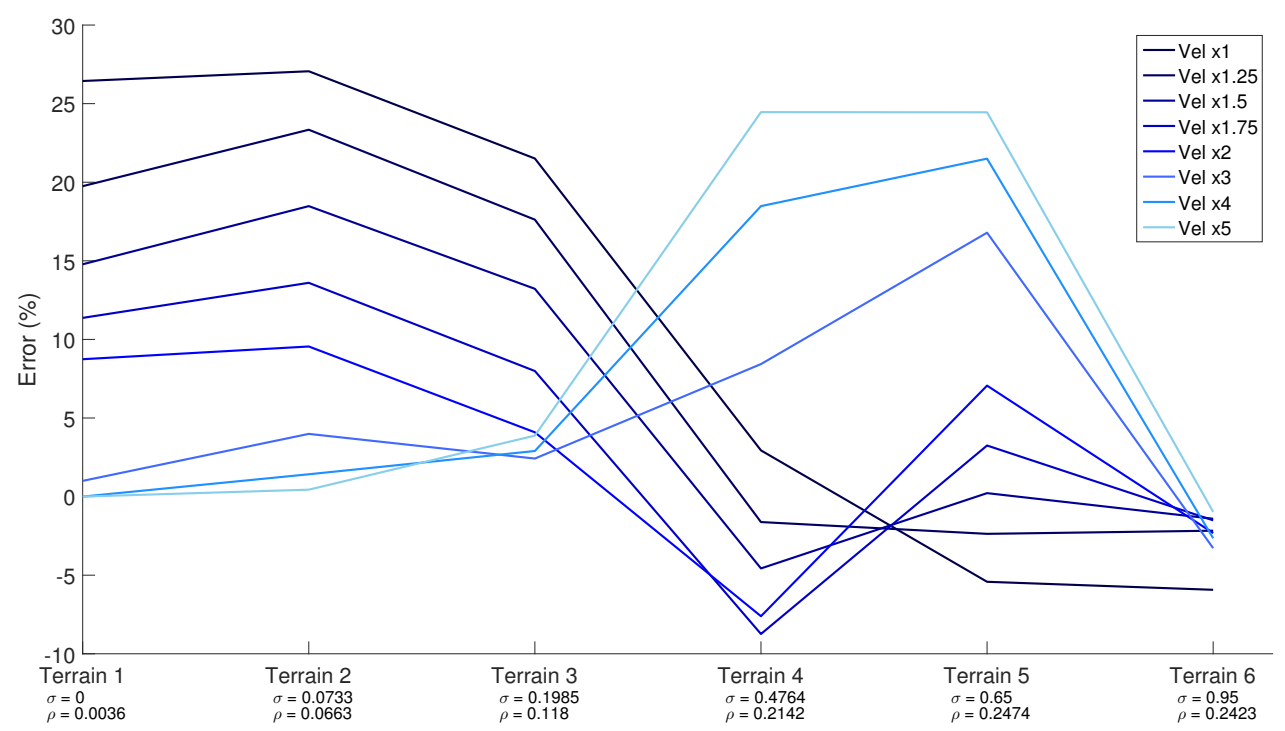

Figure 13. Errors of the slip estimator for different terrains and velocities during the acceleration steps. Analysing terrains 1 to 3, there are high errors for high velocity increments, and low errors for low velocity increments. The contrary happens for terrains 4 to 6 . A balance between errors for different parameters arise with velocity $\mathrm{x} 2$.

The speed increment during the wheel-forward step causes the rover to turn, which would be undesirable. Taking into consideration Equation (21), turning radius can be estimated during the acceleration step. Obviously, the maximum turning radius would be desirable, that is, a high turning radius allows the rover go almost straight on. Therefore, the slip estimation error would need to be minimised and the turning radius maximised. Figure 14 shows a comparative between these parameters for each speed increment. As can be appreciated, the lowest estimation error is obtained with a speed increment $x 2$, meanwhile, the highest turning radius is obtained with the minimum speed increment (1.25). 


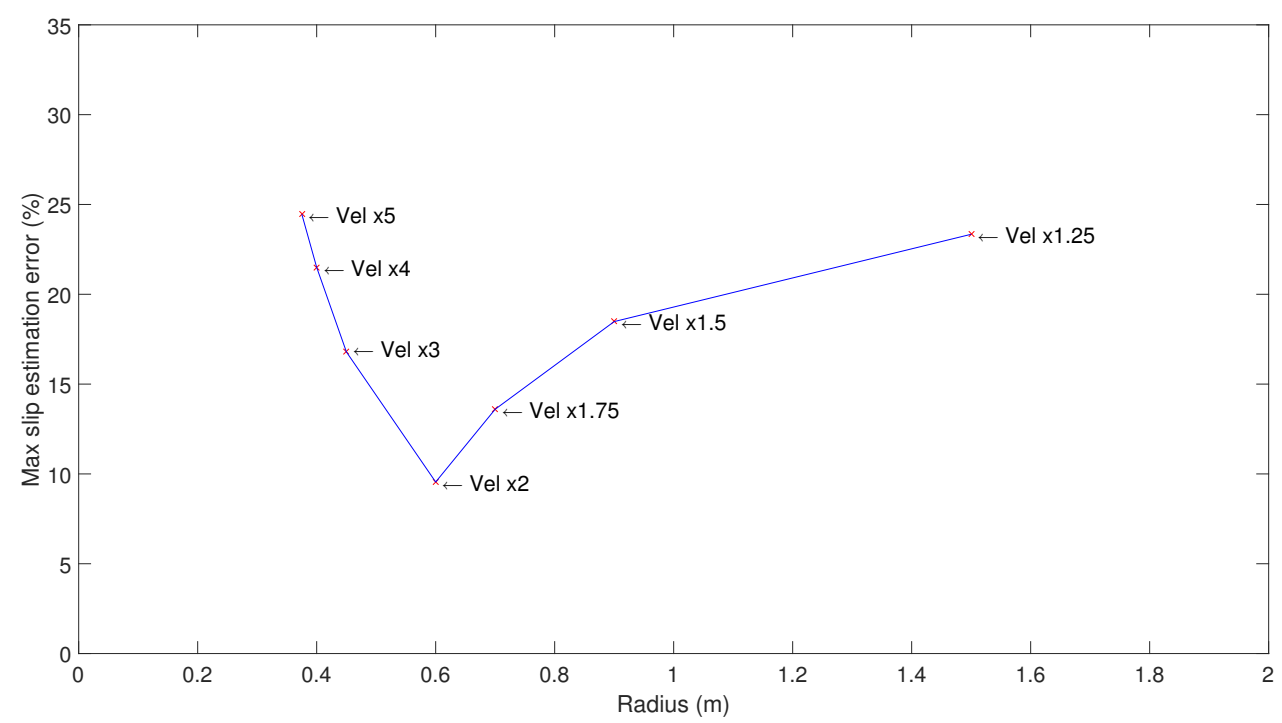

Figure 14. Representation of the parameters to be optimised, the maximum slip ratio and the rover turning radius due to the velocity increment. Based on it, the chosen velocity increment is $x 2$.

Finally, the objective is to find the best speed increment that is a balance between the estimation error and the turning radius. The speed increment $\mathrm{x} 2$ arises as the best choice, providing a maximum error of $9.55 \%$ and a turning radius of $0.6 \mathrm{~m}$.

\section{Materials}

As has been stated in this paper, two software applications were mainly used: Matlab 2015b [39] and Vortex Studio 2018a [40]. Matlab can be purchased by a license from Mathworks. It includes Matlab, as a script and command line environment, and Simulink as a block diagram programming language. In this paper, Matlab was used to generate plots and analyse obtained results, and Simulink was used to implement the locomotion modes motion planning and perform communications with Vortex Studio through the UDP interface. On the other hand, reported simulations were carried out using Vortex Studio Free Edition, a limited edition that allows basic Vortex components. It was used to implement a 3D representation with the kinematics and dynamics of the ExoTeR rover. Source code and simulation files were stored in a github repository (https:/ / github.com/spaceuma/ExoTeRModel-Locomotion-planning). By downloading them, one can reproduce the performed experiments and/or use the developed model and software to carry out new experiments.

\section{Conclusions}

This paper has analysed and evaluated the use of two locomotion modes in a reconfigurable rover. These modes are normal driving - the wheels roll and generate a drawbar pull force-and wheel walking-where a combination of wheels and legs motions are planned to move the rover forward. The kinematics and dynamics of these locomotion modes were analysed in order to obtain the corresponding equations and a wheel walking motion planner was defined. Its objective is to keep a constant rover linear velocity. On the other hand, a method for estimating the rover slip during wheel walking was defined. A simulation environment, based on Vortex Studio and Matlab-Simulink was implemented, which was used to evaluate both locomotion modes and the slip estimator. This simulation environment includes a model of the real prototype rover ExoTeR, owned by the European Space Agency.

As regards the obtained results, both locomotion modes were evaluated for 6 different terrains and slopes. As a conclusion, wheel walking is better than normal driving for terrains with low slopes, high rolling resistances and slips, normal driving is much better in the rest of the cases. 
The slip estimator has also been tested on these terrains. The main drawback of the proposed method is that the rover performs an undesirable curve due to the speed increment of half of the wheels. Thus, a comparison between the speed increment, estimation accuracy and turning radius was carried out. Results show that when doubling the speed, there is a compromise between the accuracy and the turning radius. The main limitation of the proposed work is that everything has been tested in a simulation environment since it provides ideal results that should be validated in a real environment. So, it is expected that some real experiments will be performed at the European Space Agency with the same rover presented in this paper, the ExoTeR. Moreover, more than two locomotion modes would be tested and compared in order to extend this work and provide the best one based on the defined terramechanic parameters. These extensions are proposed as future work.

Author Contributions: Conceptualization, C.J.P.d.P.M. and M.A.; methodology, Everyone; software, P.R.M. and G.J.P.D.; validation, C.J.P.d.P.M., J.R.S.I. and M.A.; writing—original draft preparation, Everyone; writing-review and editing, Everyone; supervision, C.J.P.d.P.M.; project administration, C.J.P.d.P.M. and M.A.

Funding: This research was partially funded by the European Space Agency, contract number $4000118072 / 16 / \mathrm{NL} / \mathrm{LvH} / \mathrm{gp}$, and the University of Málaga, under the project entitled: Inmersive simulation environment for robotic planetary exploration.

Acknowledgments: Authors would like to thank the Robotics and Automation Section from the European Space Agency.

Conflicts of Interest: The authors declare no conflict of interest.

\section{Abbreviations}

The following abbreviations are used in this manuscript:

$\begin{array}{ll}\text { ESA } & \text { European Space Agency } \\ \text { ESTEC } & \text { European Space Research and Technology Centre } \\ \text { GPS } & \text { Global Positioning System } \\ \text { ICR } & \text { Instant Centre of Rotation } \\ \text { ExoTeR } & \text { ExoMars Testing Rover } \\ \text { RCM } & \text { Remote Centre of Motion } \\ \text { BLM } & \text { Best Locomotion Mode }\end{array}$

\section{References}

1. Lester, D.F.; Hodges, K.V.; Anderson, R.C. Exploration telepresence: A strategy for optimizing scientific research at remote space destinations. Sci. Robot. 2017, 2, eaan4383. [CrossRef]

2. Ono, M.; Fuchs, T.J.; Steffy, A.; Maimone, M.; Yen, J. Risk-aware planetary rover operation: Autonomous terrain classification and path planning. In Proceedings of the 2015 IEEE Aerospace Conference, Big Sky, MT, USA, 7-14 March 2015; pp. 1-10.

3. Creager, C.; Johnson, K.; Plant, M.; Moreland, S.; Skonieczny, K. Push-pull locomotion for vehicle extrication. J. Terramech. 2015, 57, 71-80. [CrossRef]

4. Besseron, G.; Grand, C.; Amar, F.B.; Plumet, F.; Bidaud, P. Locomotion modes of an hybrid wheel-legged robot. In Climbing and Walking Robots; Springer: Berlin/Heidelberg, Germany, 2005; pp. 825-833.

5. Fujiwara, D.; lizuka, K. Study on bearing performance for inching worm locomotion using characteristics of wheel subsidence on loose soil. In Proceedings of the 2018 IEEE/ASME International Conference on Advanced Intelligent Mechatronics (AIM), Auckland, New Zealand, 9-12 July 2018; pp. 930-935.

6. Yeomans, B.; Saaj, C.M.; Van Winnendael, M. Walking planetary rovers-Experimental analysis and modelling of leg thrust in loose granular soils. J. Terramech. 2013, 50, 107-120. [CrossRef]

7. SunSpiral, V.; Wheeler, D.; Chavez-Clemente, D.; Mittman, D. Development and field testing of the footfall planning system for the ATHLETE robots. J. Field Robot. 2012, 29, 483-505. [CrossRef]

8. Reid, W.; Pérez-Grau, F.J.; Göktoğan, A.H.; Sukkarieh, S. Actively articulated suspension for a wheel-on-leg rover operating on a Martian analog surface. In Proceedings of the 2016 IEEE International Conference on Robotics and Automation (ICRA), Stockholm, Sweden, 16-21 May 2016; pp. 5596-5602. 
9. Cordes, F.; Dettmann, A.; Kirchner, F. Locomotion modes for a hybrid wheeled-leg planetary rover. In Proceedings of the 2011 IEEE International Conference on Robotics and Biomimetics (ROBIO), Phuket, Thailand, 7-11 December 2011; pp. 2586-2592.

10. Cordes, F.; Kirchner, F.; Babu, A. Design and field testing of a rover with an actively articulated suspension system in a Mars analog terrain. J. Field Robot. 2018, 35, 1149-1181. [CrossRef]

11. Vago, J.L.; Westall, F.; Coates, A.J.; Jaumann, R.; Korablev, O.; Ciarletti, V.; Mitrofanov, I.; Josset, J.L.; De Sanctis, M.C.; Bibring, J.P.; et al. Habitability on early Mars and the search for biosignatures with the ExoMars Rover. Astrobiology 2017, 17, 471-510. [CrossRef] [PubMed]

12. Azkarate, M.; Zwick, M.; Hidalgo-Carrio, J.; Nelen, R.; Wiese, T.; Poulakis, P.; Joudrier, L.; Visentin, G. First Experimental investigations on Wheel-Walking for improving Triple-Bogie rover locomotion performances. In Proceedings of the 13th Symposium on Advanced Space Technologies in Robotics and Automation, Noordwijk, The Netherlands, 11-13 May 2015; pp. 1-6.

13. Bauer, R.; Leung, W.; Barfoot, T. Development of a dynamic simulation tool for the Exomars rover. In Proceedings of the 8th International Symposium on Artificial Intelligence, Robotics and Automation in Space, iSAIRAS, Munich, Germany, 5-8 September 2005.

14. Thueer, T.; Krebs, A.; Siegwart, R.; Lamon, P. Performance comparison of rough-terrain robots-Simulation and hardware. J. Field Robot. 2007, 24, 251-271. [CrossRef]

15. Otsu, K.; Ono, M.; Fuchs, T.J.; Baldwin, I.; Kubota, T. Autonomous terrain classification with co-and self-training approach. IEEE Robot. Autom. Lett. 2016, 1, 814-819. [CrossRef]

16. Angelova, A.; Matthies, L.; Helmick, D.; Perona, P. Learning and prediction of slip from visual information. J. Field Robot. 2007, 24, 205-231. [CrossRef]

17. Reina, G.; Ishigami, G.; Nagatani, K.; Yoshida, K. Odometry correction using visual slip angle estimation for planetary exploration rovers. Adv. Robot. 2010, 24, 359-385. [CrossRef]

18. Brooks, C.A.; Iagnemma, K. Self-supervised terrain classification for planetary surface exploration rovers. J. Field Robot. 2012, 29, 445-468. [CrossRef]

19. Taheri, S.; Sandu, C.; Taheri, S.; Pinto, E.; Gorsich, D. A technical survey on Terramechanics models for tire-terrain interaction used in modeling and simulation of wheeled vehicles. J. Terramech. 2015, 57, 1-22. [CrossRef]

20. Ishigami, G.; Miwa, A.; Nagatani, K.; Yoshida, K. Terramechanics-based model for steering maneuver of planetary exploration rovers on loose soil. J. Field Robot. 2007, 24, 233-250. [CrossRef]

21. Ishigami, G.; Nagatani, K.; Yoshida, K. Slope traversal controls for planetary exploration rover on sandy terrain. J. Field Robot. 2009, 26, 264-286. [CrossRef]

22. Iagnemma, K.; Kang, S.; Shibly, H.; Dubowsky, S. Online terrain parameter estimation for wheeled mobile robots with application to planetary rovers. IEEE Trans. Robot. 2004, 20, 921-927. [CrossRef]

23. Yoshida, K.; Hamano, H. Motion dynamics of a rover with slip-based traction model. In Proceedings of the IEEE International Conference on Robotics and Automation, Washington, DC, USA, 11-15 May 2002; Volume 3, pp. 3155-3160.

24. Wong, J.Y. Theory of Ground Vehicles; John Wiley \& Sons: Hoboken, NJ, USA, 2008.

25. Benamar, F.; Grand, C. Quasi-static motion simulation and slip prediction of articulated planetary rovers using a kinematic approach. J. Mech. Robot. 2013, 5, 021002. [CrossRef]

26. Morales, J.; Martinez, J.L.; Mandow, A.; García-Cerezo, A.J.; Pedraza, S. Power consumption modeling of skid-steer tracked mobile robots on rigid terrain. IEEE Trans. Robot. 2009, 25, 1098-1108. [CrossRef]

27. Gillespie, T.D. Fundamentals of Vehicle Dynamics; Society of Automotive Engineers: Warrendale, PA, USA, 1992; Volume 400.

28. Wong, J.Y. Terramechanics and Off-Road Vehicles; Elsevier: Amsterdam, The Netherlands, 1989.

29. Patel, N.; Slade, R.; Clemmet, J. The ExoMars rover locomotion subsystem. J. Terramech. 2010, 47, $227-242$. [CrossRef]

30. De Canete, J.F.; Galindo, C.; Garcia-Moral, I. System Engineering and Automation: An Interactive Educational Approach; Springer Science \& Business Media: Berlin/Heidelberg, Germany, 2011.

31. Maimone, M.; Cheng, Y.; Matthies, L. Two years of visual odometry on the mars exploration rovers. J. Field Robot. 2007, 24, 169-186. [CrossRef] 
32. Ding, L.; Gao, H.; Deng, Z.; Yoshida, K.; Nagatani, K. Slip ratio for lugged wheel of planetary rover in deformable soil: Definition and estimation. In Proceedings of the IEEE/RSJ International Conference on Intelligent Robots and Systems, St. Louis, MO, USA, 10-15 October 2009; pp. 3343-3348.

33. Holzwarth, F.; Eichhorn, U. Non-contact sensors for road conditions. Sens. Actuators A Phys. 1993, 37, 121-127. [CrossRef]

34. Uno, T.; Sakai, Y.; Takagi, J.; Yamashita, T. Road surface recognition method using optical spatial filter. JSAE Rev. 1995, 2, 221.

35. Trigui, O.; Dube, Y.; Kelouwani, S.; Agbossou, K. Comparative Estimation of Electric Vehicle Rolling Resistance Coefficient in Winter Conditions. In Proceedings of the Vehicle Power and Propulsion Conference (VPPC), Hangzhou, China, 17-20 October 2016; pp. 1-6.

36. Wang, T.; Wu, Y.; Liang, J.; Han, C.; Chen, J.; Zhao, Q. Analysis and experimental kinematics of a skid-steering wheeled robot based on a laser scanner sensor. Sensors 2015, 15, 9681-9702. [CrossRef] [PubMed]

37. Azimi, A.; Holz, D.; Kövecses, J.; Angeles, J.; Teichmann, M. A multibody dynamics framework for simulation of rovers on soft terrain. J. Comput. Nonlinear Dyn. 2015, 10, 031004. [CrossRef]

38. Brooks, C.A.; Iagnemma, K.D. Self-supervised classification for planetary rover terrain sensing. In Proceedings of the 2007 IEEE Aerospace Conference, Big Sky, MT, USA, 3-10 March 2007; pp. 1-9.

39. Mathworks. Matlab Software Website. Available online: https://es.mathworks.com/products/matlab.html (accessed on 22 July 2019).

40. CM-Labs. Vortex Studio Simulation Environment Website. Available online: https://www.cm-labs.com/ vortex-studio (accessed on 29 July 2019).

(C) 2019 by the authors. Licensee MDPI, Basel, Switzerland. This article is an open access article distributed under the terms and conditions of the Creative Commons Attribution (CC BY) license (http:/ / creativecommons.org/licenses/by/4.0/). 